\title{
On the approximations of solutions to neutral SDEs with Markovian switching and jumps under non-Lipschitz conditions
}

\author{
Wei,Mao ${ }^{1}$, Xuerong, $\mathrm{Mao}^{2}$ \\ 1. School of mathematics and information technology, Jiangsu Institute of Education, \\ Nanjing, 210013, P.R.China. \\ 2. Department of Statistics and Modelling Science, University of Strathclyde, Glasgow, \\ G1 1 XH, UK.
}

\begin{abstract}
In this paper, we investigate the existence and uniqueness of solutions to neutral stochastic differential equations with Markovian switching and jumps (NSDEwMSJs) under non-Lipschitz conditions. On the other hand, we present the Euler approximate solutions for NSDEwMSJs and show that the convergence of the Euler approximate solutions to the true solutions by applying Itô formula, Bihari's lemma and Burkholder-Davis-Gundy's lemma. Some examples are provided to illustrate the main results.
\end{abstract}

Key words: Strong convergence, Neutral SDEs, Markovian switching, Poisson random measure, Non-Lipschitz conditions.

\section{Introduction}

Stochastic differential equations with Markovian switching (SDEwMSs) and stochastic differential equations with jumps (SDEwJs) have been widely used to model the phenomena arising in many branches of science and industry such fields as biology, economics, chemistry and mechanics. Qualitative theory of SDEwMSs and SDEwJs have been studied intensively for the past few years (see[1-7]). As stochastic differential equations, most SDEwMSs and SDEwJs are nonlinear and cannot be solved explicitly, so the construction of efficient computational methods is of great importance. Many mathematicians have devoted their interests to it and a substantial body work about numerical analysis for SDEwMSs and SDEwJs has been done. Here, we refer 
to Li [8], Kubilius [9], L.E.Shaikhet[10], Gardon [11], Yuan [12], Mao [13,14], Higham [15-17], Platen[18] and references therein. Recently, motivated by the theory of aeroelasticity, a class of neutral stochastic equations has also received a great deal of attention and much work has been done on neutral stochastic equations, for example, [19-24].

For above mentioned papers, most of the existing convergence theory for numerical methods requires the coefficients of SDEwMSs and neutral stochastic equations to be Lipschitz. However, the Lipschitz condition is often not met by many systems in practice. For example,

$$
d X(t)=a(r(t)) X(t) d t+b(r(t)) \sqrt{X(t)} d W(t) .
$$

did not satisfy the Lipschitz condition and we cannot apply the convergence result [12] to the Eq.(1). Therefore it is useful to establish the strong convergence of numerical method under some weak conditions. In this paper, we consider the following neutral stochastic differential equations with Markovian switching and jumps (NSDEwMSJs):

$$
\begin{cases}d[X(t)-G(r(t), X(t))]= & f(r(t), X(t)) d t+g(r(t), X(t)) d W(t) \\ & +\int_{R^{n}} h(X(t), u) \tilde{N}(d t, d u), \\ X(0)=X_{0} . & \end{cases}
$$

To the best of our knowledge, there are no literatures concerned with numerical solution of NSDEwMSJs under non-Lipschitz condition. The main aim of this paper is to prove the existence and uniqueness of the solutions to the Eq.(2) with non-Lipschitz coefficients by using a Picard type iteration; On the other hand, we will show that the Euler numerical solutions converges to the true solutions by applying Itô formula, Bihari's lemma and Burkholder-Davis-Gundy's lemma. It should be pointed out that the proof for NSDEwMSJs is certainly not a straightforward generalization of that for SDEs and SDEwMs without neutral term and jumps. Although the way of analysis follows the ideas in [14], we need to develop several new techniques to deal with the neutral term and Poisson random measure. Some known results in C.Yuan[12], X.Mao[14] are generalized to cover a class of more general NSDEsMSJs.

In Section 2, we introduce some notations and hypotheses concerning Eq.(2); In Section 3, the existence and uniqueness of the solutions to the Eq.(2) are investigated; In Section 4, we define the Euler approximate solution to NSDEwMSJs and show that the Euler approximate solution converge 
to the true solution under non-Lipschitz condition by applying some useful lemmas; In Section 5, we present two examples which illustrate the main results in this paper.

\section{Preliminaries and notations}

Let $(\Omega, \mathcal{F}, P)$ be a complete probability space with a filtration $\left(\mathcal{F}_{t}\right)_{t \geq 0}$ satisfying the usual condition, i.e. the filtration $\left(\mathcal{F}_{t}\right)$ is continuous on the right and $\left(\mathcal{F}_{0}\right)$ contains all $P$-null sets. Let $\{W(t), t \geq 0\}$ be a d-dimensional Wiener process defined on the probability space $(\Omega, \mathcal{F}, P)$ adapted to the filtration $\left(\mathcal{F}_{t}\right)_{t \geq 0}$. Let $D\left([0, T], R^{n}\right)$ denote the family of function $f$ from $[0, T] \rightarrow R^{n}$ that are right-continuous and have limits on the left, $D\left([0, T], R^{n}\right)$ is equipped with the norm $\|f\|=\sup _{0 \leq t \leq T}|f(t)|$, where $|$.$| is the Euclidean norm$ in $R^{n}$, i.e., $|x|=\sqrt{x^{\top} x}\left(x \in R^{n}\right)$. Let $T>0, \mathcal{L}^{2}\left([0, T] ; R^{n}\right)$ denote the family of all $R^{n}$-valued measurable $\left(\mathcal{F}_{t}\right)$-adapted processes $f=\{f(t)\}_{0 \leq t \leq T}$ such that $\mathrm{E} \sup _{0 \leq t \leq T}|f(t)|^{2}<\infty$.

Let $\left(R^{n}, \mathcal{B}\left(R^{n}\right)\right)$ be a measurable space and $\pi(d u)$ a $\sigma$ - finite measure on it. Let $p=p(t), t \in D_{p}$ be a stationary $\mathcal{F}_{t}$-Poisson point process on $R^{n}$ with characteristic measure $\pi$. Denote by $N(d t, d u)$ the Poisson counting measure associated with $p$, i.e.,

$$
N(t, A)=\sum_{s \in D_{p}, s \leq t} I_{A}(p(s))
$$

We refer to Ikeda [25] for the details on Poisson point process.

Let $r(t), t \geq 0$ be a right-continuous Markov chain on the probability space $(\Omega, \mathcal{F}, P)$ taking values in a finite state space $S=\{1,2 \ldots N\}$ with generator $\Gamma=\left(\gamma_{i j}\right)_{N \times N}$ given by:

$$
P(r(t+\Delta)=j \mid r(t)=i)=\left\{\begin{array}{l}
\gamma_{i j} \Delta+\circ(\Delta), \text { if } i \neq j, \\
1+\gamma_{i j} \Delta+\circ(\Delta), \text { if } i=j .
\end{array}\right.
$$

where $\Delta>0$. Here $\gamma_{i j} \geq 0$ is the transition rate from $i$ to $j, i \neq j$, While $\gamma_{i i}=-\sum_{j \neq i} \gamma_{i j}$. We assume that Markov chain $r($.$) is independent of the$ Brownian motion $W($.$) and N(d t, d u)$. It is known that almost every sample path of $r($.$) is right-continuous step function with a finite number of simple$ jumps in any finite sub-interval of $R_{+}$. 
Consider the neutral stochastic differential equations with Markovian switching and Poisson random measure:

$$
\begin{aligned}
d[X(t)-G(r(t), X(t))]= & f(r(t), X(t)) d t+g(r(t), X(t)) d W(t) \\
& +\int_{R^{n}} h(X(t), u) \tilde{N}(d t, d u), \quad 0 \leq t \leq T,
\end{aligned}
$$

with initial data $X(0)=X_{0}$. Here $f: S \times R^{n} \rightarrow R^{n}$ is the drift coefficient, $g: S \times R^{n} \rightarrow R^{n \times d}$ is the diffusion coefficient and $h: R^{n} \times R^{n} \rightarrow R^{n}$ is the jump coefficient; $W(t)$ is a d-dimensional Brownian motion and $\tilde{N}(d t, d u)$ is the compensated Poisson random measure given by

$$
\tilde{N}(d t, d u)=N(d t, d u)-\pi(d u) d t
$$

here $\pi(d u)$ is the Levy measure associated to $N$.

In this paper, we impose the following conditions on Eq.(3).

(H1) For all $x, y \in R^{n}$ and $i \in S$, there exists positive constants $\alpha_{i}$ such that

$$
\begin{aligned}
& |f(i, x)-f(i, y)|^{2} \vee|g(i, x)-g(i, y)|^{2} \\
& \vee \int_{R^{n}}|h(x, u)-h(y, u)|^{2} \pi(d u) \leq \alpha_{i} k\left(|x-y|^{2}\right),
\end{aligned}
$$

where $k($.$) is a concave nondecreasing function from R_{+}$to $R_{+}$such that $k(0)=0, k(u)>0$ for $u>0$ and $\int_{0}^{1} \frac{d u}{k(u)}=\infty$. Let $\alpha=\max _{i \in S} \alpha_{i}$.

(H2) There exist some constants $k_{i} \in(0,1)$ such that

$$
|G(i, x)-G(i, y)| \leq k_{0}|x-y|
$$

where $k_{0}=\max _{i \in S} k_{i} \in(0,1)$, and, moreover, $G(i, 0)=0$.

Remark 2.1 Let us give some concrete functions $k($.$) . Let L>0$ and $\delta \in(0,1)$ be sufficiently small. Define $k_{1}(u)=L u, u \in R_{+}$,

$$
\begin{gathered}
k_{2}(u)=\left\{\begin{array}{l}
u \log \left(1+\frac{1}{u}\right), \quad u \in[0, \delta], \\
\delta \log \left(1+\frac{1}{\delta}\right)+k_{2}^{\prime}(\delta-)(u-\delta), \quad u \in[\delta,+\infty],
\end{array}\right. \\
k_{3}(u)= \begin{cases}u \log \log \left(1+\frac{1}{u}\right), & u \in[0, \delta], \\
\delta \log \log \left(1+\frac{1}{\delta}\right)+k_{3}^{\prime}(\delta-)(u-\delta), & u \in[\delta,+\infty],\end{cases}
\end{gathered}
$$


They are all concave nondecreasing functions satisfying $\int_{0}^{1} \frac{d u}{k_{i}(u)}=+\infty(i=$ $1,2,3)$. In particular, we see clearly that if let $k(u)=L u$, then condition (4) reduce to the Lipschitz conditions. In other words, condition (4) are much weaker than the Lipschitz conditions.

\section{Existence and uniqueness of solutions}

In this section, we study the existence and uniqueness of solutions to the equation (3) under non-lipschitz condition.

In order to obtain the existence and uniqueness of solutions, we use the following Bihari's lemma which is necessary for the proof of the strong convergence of numerical solutions.

Lemma 3.1[26] Let $T>0$ and $c>0$. Let $k: R_{+} \rightarrow R_{+}$be a continuous non-decreasing function such that $k(t)>0$ for all $t>0$. Let $u($.) be a Borel measurable bounded non-negative function on $[0, T]$, and let $v($.$) be a$ non-negative integrable function on $[0, T]$. If

$$
\left.u(t) \leq c+\int_{0}^{t} v(s) k(u(s)) d s\right)
$$

for all $0 \leq t \leq T$, then

$$
u(t) \leq G^{-1}\left(G(c)+\int_{0}^{t} v(s) d s\right)
$$

holds for all such $t \in[0, T]$ that

$$
G(c)+\int_{0}^{t} v(s) d s \in \operatorname{Dom}\left(G^{-1}\right)
$$

where $G(r)=\int_{1}^{r} d s / k(s)$ on $r>0$, and $G^{-1}$ is the inverse function of $G$.

Lemma 3.2 For any $f \in \mathcal{L}^{2}\left([0, T] \times S ; R^{n}\right), g \in \mathcal{L}^{2}\left([0, T] \times S ; R^{n \times d}\right)$ and $h \in \mathcal{L}^{2}\left([0, T] \times R^{n} ; R^{n}\right)$, the following equation:

$$
\begin{gathered}
d[X(t)-G(r(t), X(t))]=\quad \\
\quad(r(t), t) d t+g(r(t), t) d W(t) \\
+\int_{R^{n}} h(t, u) \tilde{N}(d t, d u), \\
X(0)=X_{0}
\end{gathered}
$$


has a unique solution $X(t)$ on $[0, T]$ under the condition $(\mathrm{H} 2)$.

Proof: By [27], we know that there exists a sequence $\left\{\tau_{n}\right\}_{n \geq 0}$ of stopping times such that $0=\tau_{0}<\tau_{1}<\cdots<\tau_{n} \rightarrow \infty$. and $r(t)$ is constant on every interval $\left[\tau_{n}, \tau_{n+1}\right)$, that is, for every $n \geq 0$.

$$
r(t)=r\left(\tau_{n}\right), \quad \tau_{n} \leq t<\tau_{n+1} .
$$

So, we will prove that Eq.(6) has a unique solution $X(t)$ on each interval $\left[\tau_{n} \wedge T, \tau_{n+1} \wedge T\right]$. First, we consider Eq.(6) on $t \in\left[0, \tau_{1} \wedge T\right]$, which becomes

$$
\begin{aligned}
d[X(t)-G(r(0), X(t))]= & f(r(0), t) d t+g(r(0), t) d W(t) \\
& +\int_{R^{n}} h(t, u) \tilde{N}(d t, d u) .
\end{aligned}
$$

Define the operator $\Phi$

$$
\begin{aligned}
(\Phi X)(t):= & {\left[X_{0}-G\left(r(0), X_{0}\right)+G(r(0), X(t))+\int_{0}^{t} f(r(0), s) d s\right] } \\
& +\int_{0}^{t} g(r(0), s) d W(s)+\int_{0}^{t} \int_{R^{n}} h(s, u) \tilde{N}(d s, d u) \\
= & \sum_{i=1}^{3} \mathcal{G}_{i}(t) .
\end{aligned}
$$

Clearly, $\Phi X$ is $R^{n}$-valued measurable $\left\{\mathcal{F}_{t}\right\}$-adapted process. In order to obtain the existence and uniqueness of solution of Eq.(7), we give three steps as follows:

First step: we prove the mean square continuity of $\Phi$ on $[0, T]$. Let $X \in$ $R^{n}, t \in[0, T]$ and $r$ be sufficiently small, then

$$
E|(\Phi X)(t+r)-(\Phi X)(t)|^{2} \leq 3 \sum_{i=1}^{3} E\left|\mathcal{G}_{i}(t+r)-\mathcal{G}_{i}(t)\right|^{2},
$$

where

$$
\begin{aligned}
E\left|\mathcal{G}_{1}(t+r)-\mathcal{G}_{1}(t)\right|^{2} \leq & 2 E|G(r(0), X(t+r))-G(r(0), X(t))|^{2} \\
& +2 E\left|\int_{t}^{t+r} f(r(0), s) d s\right|^{2} \\
\leq & 2 k_{0}^{2} E|X(t+r)-X(t)|^{2}+2 r E \int_{t}^{t+r}|f(r(0), s)|^{2} d s .
\end{aligned}
$$


It is easy to obtain that $E\left|\mathcal{G}_{1}(t+r)-\mathcal{G}_{1}(t)\right|^{2} \rightarrow 0$, as $r \rightarrow 0$. Furthermore,

$$
\begin{aligned}
E\left|\mathcal{G}_{2}(t+r)-\mathcal{G}_{2}(t)\right|^{2} & =E\left|\int_{t}^{t+r} g(r(0), s) d W(s)\right|^{2} \\
& =E \int_{t}^{t+r}|g(r(0), s)|^{2} d s \rightarrow 0
\end{aligned}
$$

as $r \rightarrow 0$, and

$$
\begin{aligned}
E\left|\mathcal{G}_{3}(t+r)-\mathcal{G}_{3}(t)\right|^{2} & =E\left|\int_{t}^{t+r} \int_{R^{n}} h(s, u) \tilde{N}(d s, d u)\right|^{2} \\
& =E \int_{t}^{t+r} \int_{R^{n}}|h(s, u)|^{2} \pi(d u) d s \rightarrow 0
\end{aligned}
$$

as $r \rightarrow 0$. Therefore, $\Phi$ is mean square continuous on $[0, T]$.

Second step:

If $E\left(\sup _{0 \leq t \leq \tau_{1} \wedge T}|X(t)|^{2}\right)<\infty$, then we prove that $E\left(\sup _{0 \leq t \leq \tau_{1} \wedge T}|(\Phi X)(t)|^{2}\right)<\infty$.

By Hölder inequality and Doob martingale inequality, we obtain

$$
\begin{aligned}
& E\left(\sup _{0 \leq t \leq \tau_{1} \wedge T}|(\Phi X)(t)|^{2}\right) \\
\leq & 3 E \sup _{0 \leq t \leq \tau_{1} \wedge T}\left|X_{0}-G\left(r(0), X_{0}\right)+G(r(0), X(t))+\int_{0}^{t} f(r(0), s) d s\right|^{2} \\
& +3 E \sup _{0 \leq t \leq \tau_{1} \wedge T}\left|\int_{0}^{t} g(r(0), s) d W(s)\right|^{2}+3 E \sup _{0 \leq t \leq \tau_{1} \wedge T}\left|\int_{0}^{t} \int_{R^{n}} h(s, u) \tilde{N}(d s, d u)\right|^{2} \\
\leq & 9 E\left|X_{0}\right|^{2}+9 E \sup _{0 \leq t \leq \tau_{1} \wedge T}\left|G(r(0), X(t))-G\left(r(0), X_{0}\right)\right|^{2} \\
& +9 E \sup _{0 \leq t \leq \tau_{1} \wedge T}\left|\int_{0}^{t} f(r(0), s) d s\right|^{2}+12 E \int_{0}^{\tau_{1} \wedge T}|g(r(0), s)|^{2} d s \\
& +12 E \int_{0}^{\tau_{1} \wedge T} \int_{R^{n}}|h(s, u)|^{2} \pi(d u) d s \\
\leq & 9 E\left|X_{0}\right|^{2}+9 k_{0}^{2} E \sup _{0 \leq t \leq \tau_{1} \wedge T}\left|X(t)-X_{0}\right|^{2}+9\left(\tau_{1} \wedge T\right) E \int_{0}^{\tau_{1} \wedge T}|f(r(0), s)|^{2} d s \\
& +12 E \int_{0}^{\tau_{1} \wedge T}|g(r(0), s)|^{2} d s+12 E \int_{0}^{\tau_{1} \wedge T} \int_{R^{n}}|h(s, u)|^{2} \pi(d u) d s .
\end{aligned}
$$

Since $E \sup _{0 \leq t \leq T}|f(r(0), t)|^{2} \vee E \sup _{0 \leq t \leq T}|f(r(0), t)|^{2} \vee E \sup _{0 \leq t \leq T}|h(t, u)|^{2}<\infty$, so it 
follows that

$$
E\left(\sup _{0 \leq t \leq \tau_{1} \wedge T}|(\Phi X)(t)|^{2}\right)<\infty .
$$

Hence, (9) implies $\Phi$ is a operator from $\mathcal{L}^{2}\left(\left[0, \tau_{1} \wedge T\right] ; R^{n}\right)$ to itself and we conclude that $\Phi$ is well defined.

Third step: we prove that $\Phi$ has a unique fixed point. For any $X, Y \in$ $\mathcal{L}^{2}\left(\left[0, \tau_{1} \wedge T\right] ; R^{n}\right)$, we have

$$
\begin{aligned}
E \sup _{0 \leq t \leq \tau_{1} \wedge T}|(\Phi X)(t)-(\Phi Y)(t)|^{2} & =E \sup _{0 \leq t \leq \tau_{1} \wedge T}|G(r(0), X(t))-G(r(0), Y(t))|^{2} \\
& \leq k_{0}^{2} E \sup _{0 \leq t \leq \tau_{1} \wedge T}|X(t)-Y(t)|^{2}
\end{aligned}
$$

By $0 \leq k_{0}^{2}<1$, the operator $\Phi$ has a unique fixed point in $\mathcal{L}^{2}\left(\left[0, \tau_{1} \wedge T\right] ; R^{n}\right)$, i.e., there exists unique stochastic process $X=X(t)$ satisfying

$$
E \sup _{0 \leq t \leq \tau_{1} \wedge T}|X(t)-(\Phi X)(t)|^{2}=0 .
$$

So $X(t)$ is a unique solution of Eq. (6) in $\left[0, \tau_{1} \wedge T\right]$. Repeating this procedure, we will extend the solution to the entire interval $[0, T]$. The proof is complete.

Theorem 3.1. If the conditions (H1) and (H2) hold, then the Eq.(3) has a unique solution $X(t)$ on $[0, T]$.

Proof: Similarly to the discussion of lemma 3.2, we need to prove that Eq.(3) has a unique solution $X(t)$ on each interval $\left[\tau_{n} \wedge T, \tau_{n+1} \wedge T\right]$. When $t \in\left[0, \tau_{1} \wedge T\right]$, the Eq.(3) becomes

$$
\begin{aligned}
d[X(t)-G(r(0), X(t))]= & f(r(0), X(t)) d t+g(r(0), X(t)) d W(t) \\
& +\int_{R^{n}} h(X(t), u) \tilde{N}(d t, d u) .
\end{aligned}
$$

Now we prove that Eq.(3) has a unique solution $X(t)$ on $\left[0, \tau_{1} \wedge T\right]$. Let $X^{0}(t)=X_{0}, t \in\left[0, \tau_{1} \wedge T\right]$, define the following Picard sequence:

$$
\begin{aligned}
\left.X^{n}(t)-G\left(r(0), X^{n}(t)\right)\right]= & X_{0}-G\left(r(0), X_{0}\right)+\int_{0}^{t} f\left(r(0), X^{n-1}(s)\right) d s \\
& +\int_{0}^{t} g\left(r(0), X^{n-1}(s)\right) d W(s) \\
& +\int_{0}^{t} \int_{R^{n}} h\left(X^{n-1}(s), u\right) \tilde{N}(d s, d u)
\end{aligned}
$$


By lemma 3.2, we get that the Eq.(13) has a unique solution $X^{n}(t)$ on $\left[0, \tau_{1} \wedge\right.$ $T]$. Next, we are going to show that $\left\{X^{n}(t)\right\}_{n \geq 1}$ is a Cauchy sequence. It suffices to prove the following

$$
\begin{gathered}
E \sup _{0 \leq s \leq t}\left|X^{n}(s)\right|^{2} \leq C, \quad t \in\left[0, \tau_{1} \wedge T\right], \\
\limsup _{n, m \rightarrow \infty} E \max _{0 \leq t \leq \tau_{1} \wedge T}\left|X^{n}(t)-X^{m}(t)\right|^{2}=0 .
\end{gathered}
$$

By the elementary inequality,

$$
|x|^{2} \leq \frac{1}{1-C}|x-y|^{2}+\frac{1}{C}|y|^{2}, \quad C \in(0,1)
$$

We have,

$$
\begin{aligned}
E \sup _{0 \leq s \leq t}\left|X^{n}(s)\right|^{2} \leq & \frac{1}{1-k_{0}} E \sup _{0 \leq s \leq t}\left|X^{n}(s)-G\left(r(0), X^{n}(s)\right)\right|^{2} \\
& +\frac{1}{k_{0}} E \sup _{0 \leq s \leq t}\left|G\left(r(0), X^{n}(s)\right)\right|^{2} .
\end{aligned}
$$

By the Eq.(13) and the basic inequality $|a+b+c+d|^{2} \leq 4|a|^{2}+4|b|^{2}+4|c|^{2}+$ $4|d|^{2}$, it is easy to show that for $0 \leq t \leq T$,

$$
\begin{aligned}
& E \sup _{0 \leq s \leq t}\left|X^{n}(s)-G\left(r(0), X^{n}(s)\right)\right|^{2} \\
\leq & 4 E \sup _{0 \leq s \leq t}\left|X_{0}-G\left(r(0), X_{0}\right)\right|^{2} \\
& +4 E \sup _{0 \leq s \leq t}\left|\int_{0}^{s} f\left(r(0), X^{n-1}(\sigma)\right) d \sigma\right|^{2} \\
& +4 E \sup _{0 \leq s \leq t}\left|\int_{0}^{s} g\left(r(0), X^{n-1}(\sigma)\right) d W(\sigma)\right|^{2} \\
& +4 E \sup _{0 \leq s \leq t}\left|\int_{0}^{s} \int_{R^{n}} h\left(X^{n-1}(\sigma), u\right) \tilde{N}(d \sigma, d u)\right|^{2} \\
= & \mathcal{H}_{1}+\mathcal{H}_{2}+\mathcal{H}_{3}+\mathcal{H}_{4} .
\end{aligned}
$$

For the term $\mathcal{H}_{1}$ in (18), we have by (H2)

$$
\mathcal{H}_{1} \leq 8 E \sup _{0 \leq s \leq t}\left|X_{0}\right|^{2}+8 E \sup _{0 \leq s \leq t}\left|G\left(r(0), X_{0}\right)\right|^{2} \leq\left(8+8 k_{0}^{2}\right) E\left|X_{0}\right|^{2} .
$$


For the term $\mathcal{H}_{2}$ in (18), we have by $(\mathrm{H} 1)$

$$
\begin{aligned}
\mathcal{H}_{2} \leq & 4 t E \int_{0}^{t} \mid f\left(r(0),\left.X^{n-1}(s)\right|^{2} d s\right. \\
\leq & 8\left(\tau_{1} \wedge T\right) E \int_{0}^{t}\left|f\left(r(0), X^{n-1}(s)\right)-f(r(0), 0)\right|^{2} d s \\
& +8\left(\tau_{1} \wedge T\right) E \int_{0}^{t}|f(r(0), 0)|^{2} d s \\
\leq & 8\left(\tau_{1} \wedge T\right) \alpha E \int_{0}^{t} k\left(\left|X^{n-1}(s)\right|^{2}\right) d s+8\left(\tau_{1} \wedge T\right) E \int_{0}^{t}|f(r(0), 0)|^{2} d s \\
\leq & 8\left(\tau_{1} \wedge T\right) \alpha \int_{0}^{t} k\left(E\left|X^{n-1}(s)\right|^{2}\right) d s+8\left(\tau_{1} \wedge T\right) E \int_{0}^{t}|f(r(0), 0)|^{2} .(20)
\end{aligned}
$$

For the term $\mathcal{H}_{3}$ and $\mathcal{H}_{4}$ in (18), we have by (H1), Doob inequality and martingale isometries

$$
\begin{aligned}
\mathcal{H}_{3} & \leq 16 E \int_{0}^{t} \mid g\left(r(0),\left.X^{n-1}(s)\right|^{2} d s\right. \\
& \leq 32 \alpha E \int_{0}^{t} k\left(\left|X^{n-1}(s)\right|^{2}\right) d s+32 E \int_{0}^{t}|g(r(0), 0)|^{2} d s \\
& \leq 32 \alpha \int_{0}^{t} k\left(E \sup _{0 \leq \sigma \leq s}\left|X^{n-1}(\sigma)\right|^{2}\right) d s+32 E \int_{0}^{t}|g(r(0), 0)|^{2} .
\end{aligned}
$$

and

$$
\begin{aligned}
\mathcal{H}_{4} \leq & 16 E \int_{0}^{t} \int_{R^{n}}\left|h\left(X^{n-1}(s), u\right)\right|^{2} \pi(d u) d s \\
\leq & 32 E \int_{0}^{t} \int_{R^{n}}\left|h\left(X^{n-1}(s), u\right)-h(0, u)\right|^{2} \pi(d u) d s \\
& +32 E \int_{0}^{t} \int_{R^{n}}|h(0, u)|^{2} \pi(d u) d s \\
\leq & 32 \alpha \int_{0}^{t} k\left(E \sup _{0 \leq \sigma \leq s}\left|X^{n-1}(\sigma)\right|^{2}\right) d s+32 E \int_{0}^{t} \int_{R^{n}}|h(0, u)|^{2} \pi(d u) d s .
\end{aligned}
$$

Setting

$$
K=\max _{i \in S}\left\{E|f(i, 0)|^{2}, E|g(i, 0)|^{2}, E \int_{R^{n}}|h(0, u)|^{2} \pi(d u)\right\}
$$


and taking the above Eqs (19)-(22) into (18), we have

$$
\begin{aligned}
& E \sup _{0 \leq s \leq t}\left|X^{n}(s)-G\left(r(0), X^{n}(s)\right)\right|^{2} \\
\leq & \left(8+8 k_{0}^{2}\right) E\left|X_{0}\right|^{2}+\left[8\left(\tau_{1} \wedge T\right)+64\right] \alpha \int_{0}^{t} k\left(E \sup _{0 \leq \sigma \leq s}\left|X^{n-1}(\sigma)\right|^{2}\right) d s \\
& +\left[\left(\tau_{1} \wedge T\right)+64\right]\left(\tau_{1} \wedge T\right) K .
\end{aligned}
$$

Inserting (23) in (17) gives

$$
\begin{aligned}
E \sup _{0 \leq s \leq t}\left|X^{n}(s)\right|^{2} \leq & \frac{1}{1-k_{0}}\left\{\left(8+8 k_{0}^{2}\right) E\left|X_{0}\right|^{2}+\left[\left(\tau_{1} \wedge T\right)+64\right]\left(\tau_{1} \wedge T\right) K\right. \\
& \left.+\left[8\left(\tau_{1} \wedge T\right)+64\right] \int_{0}^{t} k\left(E \sup _{0 \leq \sigma \leq s}\left|X^{n-1}(\sigma)\right|^{2}\right) d s\right\} \\
& +k_{0} E \sup _{0 \leq s \leq t}\left|X^{n}(s)\right|^{2}
\end{aligned}
$$

Due to $k_{0} \in(0,1)$, we obtain that

$$
\begin{aligned}
E \sup _{0 \leq s \leq t}\left|X^{n}(s)\right|^{2} \leq & \frac{\left(8+8 k_{0}^{2}\right) E\left|X_{0}\right|^{2}+\left[\left(\tau_{1} \wedge T\right)+64\right]\left(\tau_{1} \wedge T\right) K}{\left(1-k_{0}\right)^{2}} \\
& +\frac{\left[8\left(\tau_{1} \wedge T\right)+64\right]}{\left(1-k_{0}\right)^{2}} \int_{0}^{t} k\left(E \sup _{0 \leq \sigma \leq s}\left|X^{n-1}(\sigma)\right|^{2}\right) d s .
\end{aligned}
$$

Given that $k($.$) is concave and k(0)=0$, we can find a pair of positive constants $a$ and $b$ such that

$$
k(u) \leq a u+b
$$

for all $u>0$. So we have

$$
\begin{aligned}
E \sup _{0 \leq s \leq t}\left|X^{n}(s)\right|^{2} \leq & \frac{\left(8+8 k_{0}^{2}\right) E\left|X_{0}\right|^{2}+\left[\left(\tau_{1} \wedge T\right)+64\right]\left(\tau_{1} \wedge T\right) K}{\left(1-k_{0}\right)^{2}} \\
& +\frac{8\left(\tau_{1} \wedge T\right)+64}{\left(1-k_{0}\right)^{2}} \int_{0}^{t} a E \sup _{0 \leq \sigma \leq s}\left|X^{n-1}(\sigma)\right|^{2} d s \\
& +\frac{8\left(\tau_{1} \wedge T\right)+64}{\left(1-k_{0}\right)^{2}} \int_{0}^{t} b d s \\
\leq & \frac{\left(8+8 k_{0}^{2}\right) E\left|X_{0}\right|^{2}+\left[\left(\tau_{1} \wedge T\right)+64\right]\left(\tau_{1} \wedge T\right) K}{\left(1-k_{0}\right)^{2}}
\end{aligned}
$$




$$
\begin{aligned}
& +\frac{8\left(\tau_{1} \wedge T\right)+64}{\left(1-k_{0}\right)^{2}}\left(\tau_{1} \wedge T\right) b \\
& +\frac{8\left(\tau_{1} \wedge T\right)+64}{\left(1-k_{0}\right)^{2}} \int_{0}^{t} a\left(E\left|X_{0}\right|^{2}+E \sup _{0 \leq \sigma \leq s}\left|X^{n}(\sigma)\right|^{2}\right) d s .
\end{aligned}
$$

The Gronwall inequality implies

$$
\begin{aligned}
& E\left(\sup _{0 \leq s \leq t}\left|X^{n}(s)\right|^{2}\right) \\
\leq & {\left[\frac{\left(8+8 k_{0}^{2}\right) E\left|X_{0}\right|^{2}+\left[\left(\tau_{1} \wedge T\right)+64\right]\left(\tau_{1} \wedge T\right) K}{\left(1-k_{0}\right)^{2}}\right.} \\
& \left.+\frac{8\left(\tau_{1} \wedge T\right)+64}{\left(1-k_{0}\right)^{2}}\left(\tau_{1} \wedge T\right)\left(a E\left|X_{0}\right|^{2}+b\right)\right] e^{\frac{8\left(\tau_{1} \wedge T\right)+64}{\left(1-k_{0}\right)^{2}}\left(\tau_{1} \wedge T\right)} .
\end{aligned}
$$

Then the proof of inequality (14) has been done. We turn to proving Eq.(15). Observing that

$$
\begin{aligned}
X^{n}(t)-X^{m}(t)= & G\left(r(0), X^{n}(t)\right)-G\left(r(0), X^{m}(t)\right) \\
& +\int_{0}^{t}\left[f\left(r(0), X^{n-1}(s)\right)-f\left(r(0), X^{m-1}(s)\right)\right] d s \\
& +\int_{0}^{t}\left[g\left(r(0), X^{n-1}(s)\right)-g\left(r(0), X^{m-1}(s)\right)\right] d W(s) \\
& +\int_{0}^{t} \int_{R^{n}}\left[h\left(X^{n-1}(s), u\right)-h\left(X^{m-1}(s), u\right)\right] \tilde{N}(d s, d u) .
\end{aligned}
$$

By Hölder inequality, Doob inequality, martingale isometries and (H2), we can derive that

$$
\begin{aligned}
& E \sup _{0 \leq s \leq t}\left|X^{n}(s)-X^{m}(s)\right|^{2} \\
\leq & 4 k_{0}^{2} E \sup _{0 \leq s \leq t}\left|X^{n}(s)-X^{m}(s)\right|^{2} \\
& +4\left(\tau_{1} \wedge T\right) E \int_{0}^{t}\left|f\left(r(0), X^{n-1}(s)\right)-f\left(r(0), X^{m-1}(s)\right)\right|^{2} d s \\
& +16 E \int_{0}^{t}\left|g\left(r(0), X^{n-1}(s)\right)-g\left(r(0), X^{m-1}(s)\right)\right|^{2} d s \\
& +16 E \int_{0}^{t} \int_{R^{n}}\left|h\left(X^{n-1}(s), u\right)-h\left(X^{m-1}(s), u\right)\right|^{2} \pi(d u) d s .
\end{aligned}
$$


Next, by (H1), we have

$$
\begin{aligned}
& E \sup _{0 \leq s \leq t}\left|X^{n}(s)-X^{m}(s)\right|^{2} \\
\leq & 4 k_{0}^{2} E \sup _{0 \leq s \leq t}\left|X^{n}(s)-X^{m}(s)\right|^{2} \\
& +\left[4\left(\tau_{1} \wedge T\right)+32\right] \alpha \int_{0}^{t} k\left(E \sup _{0 \leq \sigma \leq s}\left|X^{n-1}(\sigma)-X^{m-1}(\sigma)\right|^{2}\right) d s
\end{aligned}
$$

Duo to $1-4 k_{0}^{2}<1$, we obtain

$$
\begin{aligned}
& E \sup _{0 \leq s \leq t}\left|X^{n}(s)-X^{m}(s)\right|^{2} \\
\leq & \frac{\left[4\left(\tau_{1} \wedge T\right)+32\right] \alpha}{1-4 k_{0}^{2}} \int_{0}^{t} k\left(E \sup _{0 \leq \sigma \leq s}\left|X^{n-1}(\sigma)-X^{m-1}(\sigma)\right|^{2}\right) d s .
\end{aligned}
$$

By the inequality (14) and Fatou lemma, it is easily seen that

$$
\begin{aligned}
& \limsup _{n, m \rightarrow \infty} E\left(\sup _{0 \leq s \leq t}\left|X^{n}(s)-X^{m}(s)\right|^{2}\right) \\
\leq & \frac{\left[4\left(\tau_{1} \wedge T\right)+32\right] \alpha}{1-4 k_{0}^{2}} \int_{0}^{t} k\left(\limsup _{n, m \rightarrow \infty} E \sup _{0 \leq \sigma \leq s}\left|X^{n}(\sigma)-X^{m}(\sigma)\right|^{2}\right) d s .
\end{aligned}
$$

Owing to Bihari's lemma, we immediately get that

$$
\limsup _{n, m \rightarrow \infty} E\left(\sup _{0 \leq s \leq t}\left|X^{n}(s)-X^{m}(s)\right|^{2}\right)=0, \quad \text { for all } t \in\left[0, \tau_{1} \wedge T\right],
$$

Then $\left\{X^{n}(t)\right\}_{n \geq 1}$ is a Cauchy sequence under sup |.|. However, the space $D\left([0, T], R^{n}\right)$ is not a complete space under sup $|$.$| and we cannot get the$ limit of the sequence $\left\{X^{n}(t)\right\}_{n \geq 1}$. So we need to introduce a metric to make the space $D\left([0, T], R^{n}\right)$ complete. For any $X, Y \in D\left([0, T], R^{n}\right)$, we give the following metric

$$
d(X, Y)=\inf _{\lambda \in \Lambda}\left\{\sup _{0 \leq t \leq T}\left|X_{t}-Y_{\lambda(t)}\right|+\sup _{0 \leq s \leq t \leq T}\left|\log \frac{\lambda(t)-\lambda(s)}{t-s}\right|\right\}
$$

where $\Lambda=\{\lambda=\lambda(t): \lambda$ is strictly increasing, continuous on $t \in[0, T]$, such that $\lambda(0)=0, \lambda(T)=T\}$. By [28], we know that $\left(D([0, T]), R^{n}\right)$ is a complete metric space. Taking $\lambda(t)=t$, we can see $\left\{X^{n}(t)\right\}_{n \geq 1}$ is a cauchy sequence under $d(.,$.$) . Hence there exists a unique X(t) \in D\left([0, T], R^{n}\right)$ such 
that $d\left(X^{n}(),. X().\right) \rightarrow 0$ as $n \rightarrow \infty$. Denote the limit of $\left\{X^{n}(t)\right\}$ by $X(t)$, taking limits on both sides of (13) and letting $n \rightarrow \infty$, we obtain that $X(t)$ is a solution of the Eq.(12).

Now we devote to proving the uniqueness of the Eq.(12). Let $X(t)$ and $Y(t)$ be any two solutions of Eq.(12). We can prove as the same way as in proof of (29) that

$$
E \sup _{0 \leq s \leq t}|X(s)-Y(s)|^{2} \leq C \int_{0}^{t} k\left(E\left(\sup _{0 \leq \sigma \leq s}|X(\sigma)-Y(\sigma)|^{2}\right)\right) d s
$$

for all $t \in\left[0, \tau_{1} \wedge T\right]$. We get $E \sup _{0 \leq s \leq t}|X(s)-Y(s)|^{2}=0$, for all $t \in\left[0, \tau_{1} \wedge T\right]$. which implies the uniqueness.

Next, we consider the Eq.(3) on $t \in\left[\tau_{1} \wedge T, \tau_{2} \wedge T\right]$, which becomes

$$
\begin{aligned}
& d\left[X(t)-G\left(r\left(\tau_{1} \wedge T\right), X(t)\right)\right] \\
& =f\left(r\left(\tau_{1} \wedge T\right), X(t)\right) d t+g\left(r\left(\tau_{1} \wedge T\right), X(t)\right) d W(t) \\
& +\int_{R^{n}} h(X(t), u) \tilde{N}(d t, d u) .
\end{aligned}
$$

Similarly to the discussion about the existence and uniqueness of the solution of Eq.(12),we know that Eq.(31) has a unique solution $X(t)$ on $\left[\tau_{1} \wedge T, \tau_{2} \wedge\right.$ $T]$. By repeating the previous procedure, we find that Eq.(3) has a unique solution $X(t)$ on $[0, T]$. The proof is complete.

\section{Strong convergence of numerical solution under non-Lipschitz conditions}

In this section, we will show the strong convergence of the Euler approximate solution to the exact solution under non-Lipschitz condition. Before we define the Euler approximate solution for Eq.(3), we need the property of embedded discrete Markov chain. The following lemma describes this property.

Lemma 4.1[29] For $h>0$ and $n \geq 0$, then $\left\{r_{n}^{h}, n=0,1,2, \cdots\right\}$ is a discrete Markov chain with the one-step transition probability matrix

$$
P(h)=\left(P_{i j}(h)\right)_{N \times N}=e^{h \Gamma} .
$$

Given a stepsize $h>0$, the discrete Markov chain $\left\{r_{n}^{h}, n=0,1,2, \cdots\right\}$ can be simulated as follows (see C.G.Yuan[12], X.R.Mao[13]): compute the onestep transition probability matrix $P(h)=\left(P_{i j}(h)\right)_{N \times N}=e^{h \Gamma}$, Let $r(0)=i_{0}$ 
and generate a random number $\zeta_{1}$ which is uniformly distributed in $[0,1]$. If $\zeta_{1}=1$ then let $r_{1}^{h}=i_{1}=N$ or otherwise find the unique integer $i_{1} \in S$ for

$$
\sum_{j=1}^{i_{1}-1} P_{i_{0}, j}(h) \leq \zeta_{1}<\sum_{j=1}^{i_{1}} P_{i_{0}, j}(h)
$$

and let $r_{1}^{h}=i_{1}$, where we set $\sum_{i=1}^{0} P_{i_{0}, j}(h)=0$ as usual. Generate independently a new random number $\zeta_{2}$ which is again uniformly distributed in $[0,1]$. If $\zeta_{2}=1$ then let $r_{2}^{h}=i_{2}=N$ or otherwise find the unique integer $i_{2} \in S$ for

$$
\sum_{j=1}^{i_{2}-1} P_{i_{1}, j}(h) \leq \zeta_{2}<\sum_{j=1}^{i_{2}} P_{i_{1}, j}(h)
$$

and let $r_{2}^{h}=i_{2}$. Repeating this procedure a trajectory of $\left\{r_{n}^{h}, n=0,1,2, \cdots\right\}$ can be generated. This procedure can be carried out independently to obtain more trajectories.

Now we can define the Euler approximate solution of Eq.(3). For systems (3), the discrete Euler approximation on $t \in\{0, h, 2 h, \cdots\}$ is given by the iterative scheme

$$
\begin{aligned}
Y_{n+1}-G\left(r_{n+1}^{h}, Y_{n+1}\right)= & Y_{n}-G\left(r_{n}^{h}, Y_{n}\right)+f\left(r_{n}^{h}, Y_{n}\right) h \\
& +g\left(r_{n}^{h}, Y_{n}\right) \Delta W_{n}+\int_{R^{n}} h\left(Y_{n}, u\right) \tilde{N}(h, d u),
\end{aligned}
$$

Here $t_{n}=n h$ for $n \geq 0 . Y_{n} \approx X\left(t_{n}\right), \Delta W_{n}=W\left(t_{n+1}\right)-W\left(t_{n}\right)$ and $\tilde{N}(h, d u)=$ $\tilde{N}\left(t_{n+1}, d u\right)-\tilde{N}\left(t_{n}, d u\right)$. Let us introduce the following notations

$$
\bar{Y}(t)=Y_{n}, \bar{r}(t)=r_{n}^{h}
$$

for $t \in\left[t_{n}, t_{n+1}\right)$. Then we define the continuous Euler approximate solution

$$
\begin{aligned}
Y(t)= & Y_{0}+G(\bar{r}(t), \bar{Y}(t))-G\left(r(0), Y_{0}\right)+\int_{0}^{t} f(\bar{r}(s), \bar{Y}(s)) d s \\
& +\int_{0}^{t} g(\bar{r}(s), \bar{Y}(s)) d W(s)+\int_{0}^{t} \int_{R^{n}} h(\bar{Y}(s), u) \tilde{N}(d s, d u) .
\end{aligned}
$$

First of all, we give a lemma to demonstrate the existence of a solution to the Euler method (32). 
Lemma 4.2 Under the condition (H2), then the equation for the Euler method (32) can be solved uniquely for $Y_{n+1}$ given $Y_{n}$.

Proof: We may write the Eq.(32) as $Y_{n+1}=\phi\left(Y_{n+1}\right)$. By (H2), for $x, y \in R^{n}$, we have that

$$
|\phi(x)-\phi(y)|=|G(i, x)-G(i, y)| \leq K_{0}|x-y| .
$$

Hence, from the Banach contraction mapping theorem, we have that the Eq.(32) has a solutions.

We now state and prove the main theorem of this section.

Theorem 4.1 If the conditions (H1) and (H2) hold, then the Euler approximate solution (33) will converge to the exact solution of Eq.(3), i.e.,

$$
\lim _{h \rightarrow 0} E\left[\sup _{0 \leq t \leq T}|Y(t)-X(t)|^{2}\right]=0, \quad \forall T>0 .
$$

The proof of this theorem is very technical, so we present some useful Lemmas.

Lemma 4.3 Under (H1) and (H2), we get

$$
E \sup _{0 \leq t \leq T}|Y(t)|^{2} \leq C_{1}
$$

where $C_{1}=\frac{3\left(1+k_{0}^{2}\right) E\left|Y_{0}\right|^{2}+9 K T+\left(9+3 k_{1}+3 C\right) \alpha b T}{\left(1-k_{0}\right)^{2}} e^{\frac{3+3 k_{0}^{2}+\left(9+3 k_{1}+3 C\right) \alpha a}{\left(1-k_{0}\right)^{2}} T}$ dependent on $E\left|X_{0}\right|^{2}, \alpha, k_{0}$ and $T$.

Proof: By the inequality (16), we know that

$$
\begin{aligned}
E \sup _{0 \leq t \leq t_{1}}|Y(t)|^{2} \leq & \frac{1}{1-k_{0}} E \sup _{0 \leq t \leq t_{1}}|Y(t)-G(\bar{r}(t), \bar{Y}(t))|^{2} \\
& +\frac{1}{k_{0}} E \sup _{0 \leq t \leq t_{1}}|G(\bar{r}(t), \bar{Y}(t))|^{2} \\
\leq & \frac{1}{1-k_{0}} E \sup _{0 \leq t \leq t_{1}}|Y(t)-G(\bar{r}(t), \bar{Y}(t))|^{2} \\
& +k_{0} E \sup _{0 \leq t \leq t_{1}}|Y(t)|^{2} .
\end{aligned}
$$

Then

$$
E \sup _{0 \leq t \leq t_{1}}|Y(t)|^{2} \leq \frac{1}{\left(1-k_{0}\right)^{2}} E \sup _{0 \leq t \leq t_{1}}|Y(t)-G(\bar{r}(t), \bar{Y}(t))|^{2} .
$$


Applying $I t \widehat{o}^{\prime} s$ formula to $|Y(t)-G(\bar{r}(t), \bar{Y}(t))|^{2}$ yields

$$
\begin{aligned}
& |Y(t)-G(\bar{r}(t), \bar{Y}(t))|^{2} \\
= & \left|Y_{0}-G\left(r(0), Y_{0}\right)\right|^{2}+2 \int_{0}^{t}[Y(s)-G(\bar{r}(s), \bar{Y}(s))] f(\bar{r}(s), \bar{Y}(s)) d s \\
& +\int_{0}^{t}|g(\bar{r}(s), \bar{Y}(q s))|^{2} d s+\int_{0}^{t} \int_{R^{n}}|h(\bar{Y}(q s), u)|^{2} \pi(d u) d s \\
& +2 \int_{0}^{t}[Y(s)-G(\bar{r}(s), \bar{Y}(s))] g(\bar{r}(s), \bar{Y}(q s)) d W(s) \\
& +\int_{0}^{t} \int_{R^{n}}\left\{2[Y(s)-G(\bar{r}(s), \bar{Y}(s))] h(\bar{Y}(q s), u)+|h(\bar{Y}(q s), u)|^{2}\right\} \tilde{N}(d s, d u) .
\end{aligned}
$$

Thus, employing mathematical expectations and using (H2), we obtain

$$
\begin{aligned}
& E \sup _{0 \leq t \leq t_{1}}|Y(t)-G(\bar{r}(t), \bar{Y}(t))|^{2} \\
= & E\left|Y_{0}-G\left(r(0), Y_{0}\right)\right|^{2}+2 E \sup _{0 \leq t \leq t_{1}} \int_{0}^{t}[Y(s)-G(\bar{r}(s), \bar{Y}(s))] f(\bar{r}(s), \bar{Y}(s)) d s \\
& +E \sup _{0 \leq t \leq t_{1}} \int_{0}^{t}|g(\bar{r}(s), \bar{Y}(q s))|^{2} d s+E \sup _{0 \leq t \leq t_{1}} \int_{0}^{t} \int_{R^{n}}|h(\bar{Y}(q s), u)|^{2} \pi(d u) d s \\
& +2 E \sup _{0 \leq t \leq t_{1}} \int_{0}^{t}[Y(s)-G(\bar{r}(s), \bar{Y}(s))] g(\bar{r}(s), \bar{Y}(q s)) d W(s) \\
& +E \sup _{0 \leq t \leq t_{1}} \int_{0}^{t} \int_{R^{n}}\{2[Y(s)-G(\bar{r}(s), \bar{Y}(s))] h(\bar{Y}(q s), u) \\
& \left.+|h(\bar{Y}(q s), u)|^{2}\right\} N(d s, d u) \\
\leq & 2 E\left|Y_{0}\right|^{2}+2 E\left|G\left(r(0), Y_{0}\right)\right|^{2}+E \int_{0}^{t_{1}}|Y(t)-G(\bar{r}(t), \bar{Y}(t))|^{2} d s \\
& +E \int_{0}^{t_{1}}|f(\bar{r}(t), \bar{Y}(t))|^{2} d s+E \int_{0}^{t_{1}}|g(\bar{r}(t), \bar{Y}(t))|^{2} d s \\
& +E \int_{0}^{t_{1}} \int_{R^{n}}|h(\bar{Y}(t), u)|^{2} \pi(d u) d t+\mathcal{I}_{1}+\mathcal{I}_{2} .
\end{aligned}
$$

By the Burkholder-Davis-Gundy's inequality, we have

$$
\mathcal{I}_{1} \leq C E\left[\sup _{0 \leq t \leq t_{1}}|Y(t)-G(\bar{r}(t), \bar{Y}(t))|\left(\int_{0}^{t_{1}}|g(\bar{r}(t), \bar{Y}(t))|^{2} d t\right)^{\frac{1}{2}}\right]
$$




$$
\leq \frac{1}{6} E\left[\sup _{0 \leq t \leq t_{1}}|Y(t)-G(\bar{r}(t), \bar{Y}(t))|^{2}\right]+k_{1} \int_{0}^{t_{1}} E|g(\bar{r}(t), \bar{Y}(t))|^{2} d t(40)
$$

and

$$
\mathcal{I}_{2} \leq C E\left([M, M]_{t_{1}}^{\frac{1}{2}}\right),
$$

where

$$
M_{t}=\int_{0}^{t} \int_{R^{n}}\left\{2[Y(s)-G(\bar{r}(s), \bar{Y}(s))] h(\bar{Y}(s), u)+|h(\bar{Y}(s), u)|^{2}\right\} \tilde{N}(d s, d u) .
$$

By the definition of quadratic variation, we obtain

$$
\begin{aligned}
& {[M, M]_{t_{1}}^{\frac{1}{2}} } \\
= & \left\{\sum_{t \in D_{p}, t \leq t_{1}}\left(2[Y(t)-G(\bar{r}(t), \bar{Y}(t))] h(\bar{Y}(t), p(s))+|h(\bar{Y}(t), p(s))|^{2}\right)^{\frac{1}{2}}\right. \\
\leq & C\left(\sum_{t \in D_{p}, t \leq t_{1}}|Y(t)-G(\bar{r}(t), \bar{Y}(t))|^{2}|h(\bar{Y}(t), p(s))|^{2}\right)^{\frac{1}{2}} \\
& +C\left(\sum_{t \in D_{p}, t \leq t_{1}}|h(\bar{Y}(t), p(s))|^{4}\right)^{\frac{1}{2}} \\
\leq & C \sup _{0 \leq t \leq t_{1}}|Y(t)-G(\bar{r}(t), \bar{Y}(t))|\left(\sum_{t \in D_{p}, t \leq t_{1}}|h(\bar{Y}(t), p(s))|^{2}\right)^{\frac{1}{2}} \\
& +C\left(\sum_{t \in D_{p}, t \leq t_{1}}|h(\bar{Y}(t), p(s))|^{2}\right) \quad \\
\leq & \frac{1}{6} \sup _{0 \leq t \leq t_{1}}|Y(t)-G(\bar{r}(t), \bar{Y}(t))|^{2}+C\left(\sum_{t \in D_{p}, t \leq t_{1}}|h(\bar{Y}(t), p(s))|^{2}\right)
\end{aligned}
$$

So we have

$$
\begin{aligned}
\mathcal{I}_{2} & \leq \frac{1}{6} E \sup _{0 \leq t \leq t_{1}}|Y(t)-G(\bar{r}(t), \bar{Y}(t))|^{2}+C E\left(\sum_{t \in D_{p}, t \leq t_{1}}|h(\bar{Y}(t), p(s))|^{2}\right) \\
& \leq \frac{1}{6} E \sup _{0 \leq t \leq t_{1}}|Y(t)-G(\bar{r}(t), \bar{Y}(t))|^{2}+C E \int_{0}^{t_{1}} \int_{R^{n}}|h(\bar{Y}(t), u)|^{2} \pi(d u) d t .
\end{aligned}
$$


It follows from that (40) and (43), we get

$$
\begin{aligned}
& E \sup _{0 \leq t \leq t_{1}}|Y(t)-G(\bar{r}(t), \bar{Y}(t))|^{2} \\
\leq & 3 E\left|Y_{0}\right|^{2}+3 E\left|G\left(r(0), Y_{0}\right)\right|^{2}+\frac{3}{2} E \int_{0}^{t_{1}}|Y(t)-G(\bar{r}(t), \bar{Y}(t))|^{2} d s \\
& +\frac{3}{2} E \int_{0}^{t_{1}}|f(\bar{r}(t), \bar{Y}(t))|^{2} d s+\frac{3}{2}\left(1+k_{1}\right) E \int_{0}^{t_{1}}|g(\bar{r}(t), \bar{Y}(t))|^{2} d s \\
& +\frac{3}{2}(1+C) E \int_{0}^{t_{1}} \int_{R^{n}}|h(\bar{Y}(t), u)|^{2} \pi(d u) d t \\
\leq & \left.3\left(1+k_{0}^{2}\right) E\left|Y_{0}\right|^{2}+3 \int_{0}^{t_{1}} E|Y(t)|^{2} d t+3 k_{0}^{2} \int_{0}^{t} E \mid \bar{Y}(t)\right)\left.\right|^{2} d t \\
& +\left(9+3 k_{1}+3 C\right) \alpha \int_{0}^{t_{1}} k\left(E|\bar{Y}(t)|^{2} d t+3 E \int_{0}^{t_{1}}|f(\bar{r}(t), 0)|^{2} d t\right. \\
& +3 E \int_{0}^{t_{1}}|g(\bar{r}(t), 0)|^{2} d t+3 E \int_{0}^{t_{1}} \int_{R^{n}}|h(0, u)|^{2} \pi(d u) d t \\
\leq & \left.3\left(1+k_{0}^{2}\right) E\left|Y_{0}\right|^{2}+9 K T+3 \int_{0}^{t_{1}} E|Y(t)|^{2} d t+3 k_{0}^{2} \int_{0}^{t_{1}} E \mid \bar{Y}(t)\right)\left.\right|^{2} d t \\
& +\left(9+3 k_{1}+3 C\right) \alpha \int_{0}^{t_{1}} k\left(E|\bar{Y}(t)|^{2} d t .\right.
\end{aligned}
$$

Inserting (44) into (37) gives

$$
\begin{aligned}
E \sup _{0 \leq t \leq t_{1}}|Y(t)|^{2} \leq & \frac{3\left(1+k_{0}^{2}\right) E\left|Y_{0}\right|^{2}+9 K T}{\left(1-k_{0}\right)^{2}}+\frac{3+3 k_{0}^{2}}{\left(1-k_{0}\right)^{2}} \int_{0}^{t_{1}} E \sup _{0 \leq t \leq s}|Y(t)|^{2} d s \\
& +\frac{\left(9+3 k_{1}+3 C\right) \alpha}{\left(1-k_{0}\right)^{2}} \int_{0}^{t_{1}} k\left(E \sup _{0 \leq t \leq s}|Y(t)|^{2}\right) d s
\end{aligned}
$$

Given that $k($.$) is concave and k(0)=0$, we can find a pair of positive constants $a$ and $b$ such that $k(u) \leq a u+b$ for all $u>0$. So we have

$$
\begin{aligned}
E \sup _{0 \leq t \leq t_{1}}|Y(t)|^{2} \leq & \frac{3\left(1+k_{0}^{2}\right) E\left|Y_{0}\right|^{2}+9 K T+\left(9+3 k_{1}+3 C\right) \alpha b T}{\left(1-k_{0}\right)^{2}} \\
& +\frac{3+3 k_{0}^{2}+\left(9+3 k_{1}+3 C\right) \alpha a}{\left(1-k_{0}\right)^{2}} \int_{0}^{t_{1}} E \sup _{0 \leq t \leq s}|Y(t)|^{2} d s .
\end{aligned}
$$


The Gronwall inequality implies

$$
\begin{aligned}
& E \sup _{0 \leq t \leq t_{1}}|Y(t)|^{2} \\
\leq & \frac{3\left(1+k_{0}^{2}\right) E\left|Y_{0}\right|^{2}+9 K T+\left(9+3 k_{1}+3 C\right) \alpha b T}{\left(1-k_{0}\right)^{2}} e^{\frac{3+3 k_{0}^{2}+\left(9+3 k_{1}+3 C\right) \alpha a}{\left(1-k_{0}\right)^{2}} t_{1}}(.47
\end{aligned}
$$

The proof is completed.

Lemma 4.4. Under (H1), for each $t \in[0, T]$,

$$
E \sup _{0 \leq t \leq T}|Y(t)-\bar{Y}(t)|^{2} \leq C_{2} h
$$

where $C_{2}=6(T+2) k\left(C_{1}\right)+(12+6 T) K$ dependent only on $K, C_{1}$ and $T$, but independent of $h$.

Proof:For any $t \in[n h,(n+1) h)$,

$$
\begin{aligned}
\bar{Y}(t)= & Y_{n} \\
= & Y_{0}+G\left(r_{n}^{h}, Y_{n}\right)-G\left(r(0), Y_{0}\right)+\int_{0}^{n h} f(\bar{r}(s), \bar{Y}(s)) d s \\
& +\int_{0}^{n h} g(\bar{r}(s), \bar{Y}(s)) d W(s)+\int_{0}^{n h} \int_{R^{n}} h(\bar{Y}(s), u) \tilde{N}(d s, d u) .
\end{aligned}
$$

By $\bar{r}(t)=r_{n}^{h}$, thus

$$
\begin{aligned}
Y(t)-\bar{Y}(t)= & \int_{n h}^{t} f(\bar{r}(s), \bar{Y}(s)) d s+\int_{n h}^{t} g(\bar{r}(s), \bar{Y}(s)) d W(s) \\
& +\int_{n h}^{t} \int_{R^{n}} h(\bar{Y}(s), u) \tilde{N}(d s, d u) .
\end{aligned}
$$

Using the basic inequality $|a+b+c|^{2} \leq 3|a|^{2}+3|b|^{2}+3|c|^{2}$, martingale isometries and $\left(H_{1}\right)$, it follows that

$$
\begin{aligned}
& E \sup _{0 \leq t \leq T}|Y(t)-\bar{Y}(t)|^{2} \\
\leq & 3 h E \int_{0}^{T}|f(\bar{r}(s), \bar{Y}(s))|^{2} d s \\
& +3 E \sup _{0 \leq t \leq T} \max _{n=0,1,2, \cdot, N-1}\left|\int_{n h}^{t} g(\bar{r}(s), \bar{Y}(q s)) d W(s)\right|^{2}
\end{aligned}
$$




$$
\begin{aligned}
& +3 E \sup _{0 \leq t \leq T} \max _{n=0,1,2, \cdot, N-1}\left|\int_{n h}^{t} \int_{R^{n}} h(\bar{Y}(q s), u) \tilde{N}(d s, d u)\right|^{2} \\
\leq & 6 h E \int_{0}^{T}|f(\bar{r}(s), \bar{Y}(s))-f(\bar{r}(s), 0)|^{2} d s \\
& +6 h E \int_{0}^{T}|f(\bar{r}(s), 0)|^{2} d s+3 \max _{n=0,1,2, \cdot, N-1} E \int_{n h}^{(n+1) h}|g(\bar{r}(s), \bar{Y}(q s))|^{2} d s \\
& +3 \max _{n=0,1,2, \cdot, N-1} E \int_{n h}^{(n+1) h} \int_{R^{n}}|h(\bar{Y}(s), u)|^{2} \pi(d u) d s \\
\leq & 6 h E \int_{0}^{T} k\left(|\bar{Y}(s)|^{2}\right) d s+12 E \int_{n h}^{(n+1) h} k\left(E|\bar{Y}(s)|^{2}\right) d s+(12+6 T) h K \\
\leq & 6 h \int_{0}^{T} k\left(\sup _{0 \leq t \leq s} E|\bar{Y}(t)|^{2}\right) d t+12 \int_{n h}^{(n+1) h} k\left(\sup _{0 \leq t \leq s} E|\bar{Y}(t)|^{2}\right) d t+(12+6 T) h K \\
\leq & 6(T+2) k\left(C_{1}\right) h+(12+6 T) h K \\
\leq & C_{2} h .
\end{aligned}
$$

The proof is completed.

Lemma 4.5 Under condition (H2), then

$$
E \sup _{0 \leq t \leq T}|G(r(t), \bar{Y}(t))-G(\bar{r}(t), \bar{Y}(t))|^{2} \leq C_{3} h,
$$

where $C_{3}$ is a constant which is independent of $h$.

Proof:Let $n=[T / h]$ be the integer part of $T / h$. Then

$$
\begin{aligned}
& E \sup _{0 \leq t \leq T}|G(r(t), \bar{Y}(t))-G(\bar{r}(t), \bar{Y}(t))|^{2} \\
\leq & \max _{0 \leq k \leq n} E\left[\sup _{t \in[k h,(k+1) h)}|G(r(t), \bar{Y}(t))-G(\bar{r}(t), \bar{Y}(t))|^{2}\right] .
\end{aligned}
$$

Let $I_{G}$ be the indicator function of the set $G$. With these notations we derive, using (H2), that

$$
\begin{aligned}
& E \sup _{0 \leq t \leq T}|G(r(t), \bar{Y}(t))-G(\bar{r}(t), \bar{Y}(t))|^{2} \\
\leq & 2 \max _{0 \leq k \leq n} E\left[\sup _{t \in[k h,(k+1) h)}|G(r(t), \bar{Y}(t))-G(\bar{r}(t), \bar{Y}(t))|^{2} I_{\left\{r(t) \neq r\left(t_{k}\right)\right\}}\right] \\
\leq & 4 \max _{0 \leq k \leq n} E\left[\sup _{t \in[k h,(k+1) h)}\left(|G(r(t), \bar{Y}(t))|^{2}+|G(\bar{r}(t), \bar{Y}(t))|^{2}\right) I_{\left\{r(t) \neq r\left(t_{k}\right)\right\}}\right] \\
\leq & 8 k_{0}^{2} \max _{0 \leq k \leq n} E\left[\left(\sup _{t \in[k h,(k+1) h)}|\bar{Y}(t)|^{2}\right) I_{\left\{r(t) \neq r\left(t_{k}\right)\right\}}\right]
\end{aligned}
$$




$$
\begin{aligned}
& =8 k_{0}^{2} \max _{0 \leq k \leq n} E\left\{E\left[\left(\sup _{t \in[k h,(k+1) h)}|\bar{Y}(t)|^{2}\right) I_{\left\{r(t) \neq r\left(t_{k}\right)\right\}} \mid r\left(t_{k}\right)\right]\right\} \\
& \leq 8 k_{0}^{2} \max _{0 \leq k \leq n} E\left[E\left[\left(\sup _{t \in[k h,(k+1) h)}|\bar{Y}(t)|^{2}\right) \mid r\left(t_{k}\right)\right]\right] E\left[I_{\left\{r(t) \neq r\left(t_{k}\right)\right\}} \mid r\left(t_{k}\right)\right],
\end{aligned}
$$

where in the last step we use the fact that $Y_{n}$ and $I_{\left\{r(s) \neq r\left(t_{k}\right)\right\}}$ are conditionlly independent with respect to the $\sigma$-algebra generated by $r\left(t_{k}\right)$. Using the Markov property,

$$
\begin{aligned}
E\left(I_{\left\{r(t) \neq r\left(t_{k}\right)\right\}} \mid r\left(t_{k}\right)\right) & =\sum_{i \in S} I_{\left\{r\left(t_{k}\right)=i\right\}} P\left(r(s) \neq i \mid r\left(t_{k}\right)=i\right) \\
& =\sum_{i \in S} I_{\left\{r\left(t_{k}\right)=i\right\}} \sum_{j \neq i}\left(\gamma_{i j}\left(s-t_{k}\right)+\circ\left(s-t_{k}\right)\right) \\
& \leq\left(\max _{1 \leq i \leq N}\left(-\gamma_{i i}\right) h+\circ(h)\right) \sum_{i \in S} I_{\left\{r\left(t_{k}\right)=i\right\}} \\
& \leq C^{\prime} h+\circ(h) .
\end{aligned}
$$

Lemma 4.3 yields,

$$
\begin{aligned}
& E \sup _{0 \leq t \leq T}|G(r(t), \bar{Y}(t))-G(\bar{r}(t), \bar{Y}(t))|^{2} \\
\leq & 8 k_{0}^{2} C^{\prime} h \max _{0 \leq k \leq n} E\left[E\left[\left(\sup _{t \in[k h,(k+1) h)}|\bar{Y}(t)|^{2}\right) \mid r\left(t_{k}\right)\right]\right] \\
\leq & 8 k_{0}^{2} C^{\prime} h \max _{0 \leq k \leq n} E\left(\sup _{0 \leq t \leq T}|Y(t)|^{2}\right) \\
\leq & 8 k_{0}^{2} C_{1} C^{\prime} h \\
\leq & C_{3} h .
\end{aligned}
$$

The proof is completed.

Lemma 4.6 Under condition (H1), then

$$
\begin{aligned}
& E \int_{0}^{T}|f(\bar{r}(s), \bar{Y}(s))-f(r(s), \bar{Y}(s))|^{2} d s \\
& \vee E \int_{0}^{T}|g(\bar{r}(s), \bar{Y}(s))-g(r(s), \bar{Y}(s))|^{2} d s \leq C_{4} h,
\end{aligned}
$$

where $C_{4}$ is a constant which is independent of $h$.

Proof. The proof is similar to that of lemma 3 in [14], and we thus omit 
here.

Proof of Theorem 4.1. By the definitions of $X(t)$ and $Y(t)$, we have

$$
\begin{aligned}
& X(t)-Y(t) \\
= & G(r(t), X(t))-G(\bar{r}(t), \bar{Y}(t))+\int_{0}^{t}[f(r(s), X(s))-f(\bar{r}(s), \bar{Y}(s))] d s \\
& +\int_{0}^{t}[g(r(s), X(s))-g(\bar{r}(s), \bar{Y}(s))] d W(s) \\
& +\int_{0}^{t} \int_{R^{n}}[h(Y(s), u)-h(\bar{Y}(s), u)] \tilde{N}(d s, d u)
\end{aligned}
$$

By the inequality (16), we get

$$
\begin{aligned}
& E \sup _{0 \leq t \leq t_{1}}|X(t)-Y(t)|^{2} \\
\leq & \frac{1}{1-k_{0}} E \sup _{0 \leq t \leq t_{1}}|[X(t)-Y(t)]-[G(r(t), X(t))-G(\bar{r}(t), \bar{Y}(t))]|^{2} \\
& +\frac{1}{k_{0}} E \sup _{0 \leq t \leq t_{1}}|G(r(t), X(t))-G(\bar{r}(t), \bar{Y}(t))|^{2} .
\end{aligned}
$$

Applying Itố $s$ formula to $|[X(t)-Y(t)]-[G(r(t), X(t))-G(\bar{r}(t), \bar{Y}(t))]|^{2}$ and taking expectations, yields

$$
\begin{aligned}
& E \sup _{0 \leq t \leq t_{1}}|[X(t)-Y(t)]-[G(r(t), X(t))-G(\bar{r}(t), \bar{Y}(t))]|^{2} \\
= & 2 E \int_{0}^{t_{1}}\{[X(s)-Y(s)]-[G(r(s), X(s))-G(\bar{r}(s), \bar{Y}(s))]\} \\
& {[f(r(s), X(s))-f(\bar{r}(s), \bar{Y}(s))] d s } \\
& +E \int_{0}^{t_{1}}|g(r(s), X(s))-g(\bar{r}(s), \bar{Y}(s))|^{2} d s \\
& +E \int_{0}^{t_{1}} \int_{R^{n}}|h(Y(s), u)-h(\bar{Y}(s), u)|^{2} \pi(d u) d s \\
& +2 E \sup _{0 \leq t \leq t_{1}} \int_{0}^{t}\{[X(s)-Y(s)]-[G(r(s), X(s))-G(\bar{r}(s), \bar{Y}(s))]\} \\
& {[g(r(s), X(s))-g(\bar{r}(s), \bar{Y}(s))] d W(s) } \\
& +2 E \sup _{0 \leq t \leq t_{1}} \int_{0}^{t} \int_{R^{n}}\{\{[X(s)-Y(s)]-[G(r(s), X(s))-G(\bar{r}(s), \bar{Y}(s))]\}
\end{aligned}
$$




$$
\begin{aligned}
& {\left.[h(X(s), u)-h(\bar{Y}(s), u)]+|h(X(s), u)-h(\bar{Y}(s), u)|^{2}\right\} \tilde{N}(d s, d u) } \\
= & \mathcal{J}_{1}+\mathcal{J}_{2}+\mathcal{J}_{3}+\mathcal{J}_{4}+\mathcal{J}_{5}
\end{aligned}
$$

Where

$$
\begin{aligned}
\mathcal{J}_{1} \leq & E \int_{0}^{t_{1}}|[X(s)-Y(s)]-[G(r(s), X(s))-G(\bar{r}(s), \bar{Y}(s))]|^{2} d s \\
& +E \int_{0}^{t_{1}}|f(r(s), X(s))-f(\bar{r}(s), \bar{Y}(s))|^{2} d s \\
\leq & E \int_{0}^{t_{1}}|X(s)-Y(s)|^{2} d s+E \int_{0}^{t_{1}}|G(r(s), X(s))-G(\bar{r}(s), \bar{Y}(s))|^{2} d s \\
& +E \int_{0}^{t_{1}}|f(r(s), X(s))-f(\bar{r}(s), \bar{Y}(s))|^{2} d s \\
= & E \int_{0}^{t_{1}}|X(s)-Y(s)|^{2} d s+\mathcal{L}_{1}+\mathcal{L}_{2} .
\end{aligned}
$$

Taking into accounts lemmas 4.4 and 4.5, we have

$$
\begin{aligned}
\mathcal{L}_{1} \leq & 2 E \int_{0}^{t_{1}}|G(r(s), X(s))-G(r(s), \bar{Y}(s))|^{2} d s \\
& +2 E \int_{0}^{t_{1}}|G(r(s), \bar{Y}(s))-G(\bar{r}(s), \bar{Y}(s))|^{2} d s \\
\leq & 2 k_{0}^{2} E \int_{0}^{t_{1}}|X(s)-\bar{Y}(s)|^{2} d s+2 C_{3} T h \\
\leq & 4 k_{0}^{2} E \int_{0}^{t_{1}}|X(s)-Y(s)|^{2} d s+4 k_{0}^{2} E \int_{0}^{t_{1}}|Y(s)-\bar{Y}(s)|^{2} d s+2 C_{3} T h \\
\leq & 4 k_{0}^{2} E \int_{0}^{t_{1}}|X(s)-Y(s)|^{2} d s+2\left(2 k_{0}^{2} C_{2}+C_{3}\right) T h .
\end{aligned}
$$

In view of $(\mathrm{H} 1)$, we obtain from lemma 4.6 that

$$
\begin{aligned}
\mathcal{L}_{2} \leq & 2 E \int_{0}^{t_{1}}|f(r(s), X(s))-f(r(s), \bar{Y}(s))|^{2} d s \\
& +2 E \int_{0}^{t_{1}}|f(r(s), \bar{Y}(s))-f(\bar{r}(s), \bar{Y}(s))|^{2} d s \\
\leq & 2 \alpha E \int_{0}^{t_{1}} k\left(|X(s)-\bar{Y}(s)|^{2}\right) d s+2 C_{4} T h
\end{aligned}
$$




$$
\begin{aligned}
& \leq 2 \alpha E \int_{0}^{t_{1}} k\left(2|X(s)-Y(s)|^{2}+2|Y(s)-\bar{Y}(s)|^{2}\right) d s+2 C_{4} T h \\
& \leq 4 \alpha E \int_{0}^{t_{1}} k\left(|X(s)-Y(s)|^{2}\right) d s+4 \alpha E \int_{0}^{t_{1}} k\left(|Y(s)-\bar{Y}(s)|^{2}\right) d s+2 C_{4} T h \\
& \leq 4 \alpha \int_{0}^{t_{1}} k\left(E|X(s)-Y(s)|^{2}\right) d s+4 \alpha \int_{0}^{t_{1}} k\left(E|Y(s)-\bar{Y}(s)|^{2}\right) d s+2 C_{4} T h \\
& \leq 4 \alpha \int_{0}^{t_{1}} k\left(E|X(s)-Y(s)|^{2}\right) d s+4 \alpha C_{2} T k(h)+2 C_{4} T h .
\end{aligned}
$$

Substituting (59) and (60) into (58) yields that

$$
\begin{aligned}
\mathcal{J}_{1} \leq & \left(1+4 k_{0}^{2}\right) E \int_{0}^{t_{1}}|X(s)-Y(s)|^{2} d s+4 \alpha \int_{0}^{t_{1}} k\left(E|X(s)-Y(s)|^{2}\right) d s \\
& +2\left(2 k_{0}^{2} C_{2}+C_{3}\right) T h+4 \alpha C_{2} T k(h)+2 C_{4} T h .
\end{aligned}
$$

Following from the proof (60), we can obtain that

$$
\mathcal{J}_{2} \leq 4 \alpha \int_{0}^{t_{1}} k\left(E|X(s)-Y(s)|^{2}\right) d s+4 \alpha C_{2} T k(h)+2 C_{4} T h .
$$

and

$$
\mathcal{J}_{3} \leq 2 \alpha \int_{0}^{t_{1}} k\left(E|X(s)-Y(s)|^{2}\right) d s+2 \alpha C_{2} T k(h) .
$$

Applying the Burkholder-Davis-Gundy's inequality and taking into account lemmas 4.5, 4.6, we have

$$
\begin{aligned}
\mathcal{J}_{4} \leq & C E\left[\sup _{0 \leq t \leq t_{1}}|[X(t)-Y(t)]-[G(r(t), X(t))-G(\bar{r}(t), \bar{Y}(t))]|\right. \\
& \left.\left(\int_{0}^{t_{1}}|g(r(t), X(t))-g(\bar{r}(t), \bar{Y}(t))|^{2} d t\right)^{\frac{1}{2}}\right] \\
\leq & \frac{1}{4} E\left[\sup _{0 \leq t \leq t_{1}}|[X(t)-Y(t)]-[G(r(t), X(t))-G(\bar{r}(t), \bar{Y}(t))]|^{2}\right] \\
& +k_{2} \int_{0}^{t_{1}} E|g(r(t), X(t))-g(\bar{r}(t), \bar{Y}(t))|^{2} d t \\
\leq & \frac{1}{4} E\left[\sup _{0 \leq t \leq t_{1}}|[X(t)-Y(t)]-[G(r(t), X(t))-G(\bar{r}(t), \bar{Y}(t))]|^{2}\right] \\
& +4 \alpha k_{2} \int_{0}^{t_{1}} k\left(E|X(s)-Y(s)|^{2}\right) d s+4 \alpha C_{2} k_{2} T k(h)+2 C_{4} k_{2} T h .
\end{aligned}
$$


and

$$
\begin{aligned}
\mathcal{J}_{5} \leq & \frac{1}{4} E \sup _{0 \leq t \leq t_{1}}|[X(t)-Y(t)]-[G(r(t), X(t))-G(\bar{r}(t), \bar{Y}(t))]|^{2} \\
& +C E\left(\sum_{t \in D_{p}, t \leq t_{1}}|h(X(s), u)-h(\bar{Y}(s), p(s))|^{2}\right) \\
\leq & \frac{1}{4} E \sup _{0 \leq t \leq t_{1}}|[X(t)-Y(t)]-[G(r(t), X(t))-G(\bar{r}(t), \bar{Y}(t))]|^{2} \\
& +C E \int_{0}^{t_{1}} \int_{R^{n}}|h(X(s), u)-h(\bar{Y}(s), u)|^{2} \pi(d u) d t \\
\leq & \frac{1}{4} E \sup _{0 \leq t \leq t_{1}}|[X(t)-Y(t)]-[G(r(t), X(t))-G(\bar{r}(t), \bar{Y}(t))]|^{2} \\
& +2 \alpha C \int_{0}^{t_{1}} k\left(E|X(s)-Y(s)|^{2}\right) d s+2 \alpha C_{2} C T k(h) .
\end{aligned}
$$

Putting (61)-(65) into (57) yields that

$$
\begin{aligned}
& E \sup _{0 \leq t \leq t_{1}}|[X(t)-Y(t)]-[G(r(t), X(t))-G(\bar{r}(t), \bar{Y}(t))]|^{2} \\
\leq & 2\left(1+4 k_{0}^{2}\right) E \int_{0}^{t_{1}}|X(s)-Y(s)|^{2} d s \\
& +4\left(5+2 k_{2}+C\right) \alpha \int_{0}^{t_{1}} k\left(E|X(s)-Y(s)|^{2}\right) d s \\
& +4\left[5 \alpha C_{2} T+2 \alpha C_{2} k_{2} T+\alpha C_{2} C T\right] k(h) \\
& +4\left[\left(2 k_{0}^{2} C_{2}+C_{3}\right) T+2 C_{4} T+C_{4} k_{2} T\right] h .
\end{aligned}
$$

On the other hand, it follows from (59) that

$$
\begin{aligned}
& E \sup _{0 \leq t \leq t_{1}}|G(r(t), X(t))-G(\bar{r}(t), \bar{Y}(t))|^{2} \\
\leq & 4 k_{0}^{2} E \sup _{0 \leq t \leq t_{1}}|X(t)-Y(t)|^{2}+\left(4 k_{0}^{2} C_{2}+2 C_{3}\right) h .
\end{aligned}
$$

Finally, taking (66) and (67) into (56), we obtain

$$
\begin{aligned}
E \sup _{0 \leq t \leq t_{1}}|X(t)-Y(t)|^{2} \leq & \frac{2\left(1+4 k_{0}^{2}\right)}{\left(1-4 k_{0}\right)\left(1-k_{0}\right)} E \int_{0}^{t_{1}}|X(s)-Y(s)|^{2} d s \\
& +\frac{4\left(5+2 k_{2}+C\right) \alpha}{\left(1-4 k_{0}\right)\left(1-k_{0}\right)} \int_{0}^{t_{1}} k\left(E|X(s)-Y(s)|^{2}\right) d s
\end{aligned}
$$




$$
\begin{aligned}
& +\frac{4\left[5 \alpha C_{2} T+2 \alpha C_{2} k_{2} T+\alpha C_{2} C T\right]}{\left(1-4 k_{0}\right)\left(1-k_{0}\right)} k(h) \\
& +\left\{\frac{4\left[\left(2 k_{0}^{2} C_{2}+C_{3}\right) T+2 C_{4} T+C_{4} k_{2} T\right]}{\left(1-4 k_{0}\right)\left(1-k_{0}\right)}\right. \\
& \left.+\frac{4 k_{0} C_{2}}{1-4 k_{0}}+\frac{2 C_{3}}{\left(1-4 k_{0}\right)\left(1-k_{0}\right)}\right\} h .
\end{aligned}
$$

By letting

$$
\begin{gathered}
M_{1}=\max \left\{\frac{2\left(1+4 k_{0}^{2}\right)}{\left(1-4 k_{0}\right)\left(1-k_{0}\right)}, \frac{4\left(5+2 k_{2}+C\right) \alpha}{\left(1-4 k_{0}\right)\left(1-k_{0}\right)}\right\}, \\
M_{2}=\max \left\{\frac{4\left[5 \alpha C_{2} T+2 \alpha C_{2} k_{2} T+\alpha C_{2} C T\right]}{\left(1-4 k_{0}\right)\left(1-k_{0}\right)},\right. \\
\left.\frac{4\left[\left(2 k_{0}^{2} C_{2}+C_{3}\right) T+2 C_{4} T+C_{4} k_{2} T\right]}{\left(1-4 k_{0}\right)\left(1-k_{0}\right)}+\frac{4 k_{0} C_{2}}{1-4 k_{0}}+\frac{2 C_{3}}{\left(1-4 k_{0}\right)\left(1-k_{0}\right)}\right\} .
\end{gathered}
$$

we have

$$
\begin{aligned}
& E \sup _{0 \leq t \leq t_{1}}|X(t)-Y(t)|^{2} \\
\leq & M_{1} \int_{0}^{t_{1}}\left[E \sup _{0 \leq t \leq s}|X(t)-Y(t)|^{2}+k\left(E \sup _{0 \leq t \leq s}|X(t)-Y(t)|^{2}\right)\right] d s \\
& +M_{2}[k(h)+h] .
\end{aligned}
$$

Let $\tilde{k}(u)=u+k(u)$, we obtain that

$$
E \sup _{0 \leq t \leq t_{1}}|X(t)-Y(t)|^{2} \leq M_{1} \int_{0}^{t} \tilde{k}\left(E \sup _{0 \leq t \leq s}|X(t)-Y(t)|^{2}\right) d s+M_{2} \tilde{k}(h) .
$$

Obviously, $\tilde{k}(0)=0$. Since $k($.$) is a concave function and k(0)=0$, we have $k(u) \geq k(1) u$, for $0 \leq u \leq 1$. So

$$
\int_{0}^{1} \frac{d u}{\tilde{k}(u)}=\int_{0}^{1} \frac{d u}{u+k(u)} \geq \frac{k(1)}{k(1)+1} \int_{0}^{1} \frac{d u}{k(u)}=\infty .
$$


By applying the Bihari inequality, it follows that

$$
E\left(\sup _{0 \leq s \leq t}|X(s)-Y(s)|^{2}\right) \leq G^{-1}\left[G\left(M_{2} \tilde{k}(h)\right)+M_{1} t\right] .
$$

Note that when $h \rightarrow 0$, then $M_{2} \tilde{k}(h) \rightarrow 0$. Recalling the condition $\int_{0}^{1} d u / \tilde{k}(u)=$ $\infty$ we have $G\left(M_{2} \tilde{k}(h)\right) \rightarrow-\infty$, and $G\left(M_{2} \tilde{k}(h)\right)+M_{1} t \rightarrow-\infty$, So we get

$$
G^{-1}\left[G\left(M_{2} \tilde{k}(h)\right)+M_{1} t\right] \rightarrow 0 .
$$

We therefore have

$$
\lim _{h \rightarrow 0} E\left(\sup _{0 \leq t \leq t_{1}}|X(t)-Y(t)|^{2}\right) \leq \lim _{h \rightarrow 0} G^{-1}\left[G\left(M_{2} \tilde{k}(h)\right)+M_{1} t\right]=0 .
$$

The proof of Theorem 4.1 is now complete.

Remark 4.1 When $G=0, h=0$, Eq.(3) reduces to

$$
d X(t)=f(r(t), X(t)) d t+g(r(t), X(t)) d W(t)
$$

which was recently studied in X.R.Mao etc[14]. Hence, Theorem 4.1 in this paper is a generalization of Theorem of [14].

Remark 4.2 If $k(u)=L u$ in $(4)(u \geq 0, L \geq 0)$, then condition (H1) implies the global Lipschitz condition.

The following corollary is an immediate consequence of Theorem 4.1 and it reveals the order of the convergence.

Corollary 4.1. Under condition (H2) and the global Lipschitz condition, i.e, there exist a positive constants $L>0$ such that for all $x, y \in R^{n}$ and $i \in S, u \in R^{n}$,

$$
\begin{aligned}
& |f(i, x)-f(i, y)|^{2} \vee|g(i, x)-g(i, y)|^{2} \\
& \vee \int_{R^{n}}|h(x, u)-h(y, u)|^{2} \pi(d u) \leq L|x-y|^{2},
\end{aligned}
$$

Then the numerical solution (33) will converge to the exact solution of Eq.(3) in the mean-square sense with order $\frac{1}{2}$, i.e, there exists a positive constant $C$ such that

$$
E\left[\sup _{0 \leq t \leq T}|Y(t)-X(t)|^{2}\right] \leq C h,
$$

where $C$ is a positive constant which is independent of $h$.

Remark 4.3 Even if Eq.(3) satisfies the Lipschitz condition (74), the Corollary 4.1 is a new result. When $G=0, h \equiv 0$, the Corollary 4.1 reduces to Theorem 3.1 of [12], so we generalize and improve the corresponding results given in [12]. 


\section{Remarks and examples}

In this section we present two examples which illustrates the main results.

Example 5.1 Let $w(t)$ be a scalar Brownian motion. Let $r(t)$ be a rightcontinuous Markov chain taking values in $S=\{1,2\}$ with the generator

$$
\Gamma=\left(\gamma_{i j}\right)_{2 \times 2}=\left[\begin{array}{rr}
-1 & 1 \\
1 & -1
\end{array}\right]
$$

Of course $w(t)$ and $r(t)$ are assumed to be independent. Consider the following semi-linear NSDEs with Markovian switching of the form

$$
d[x(t)-k x(t)]=a(r(t)) x(t) d t+b(r(t))|x(t)|^{\beta} d w_{t},
$$

here $G(r(t), x(t))=k x(t), k \in(0,1), a(1)=-1, a(2)=2, b(1)=0.5$, $b(2)=1$. The equation (76) can be regarded as the result of the two equations

$$
d[x(t)-k x(t)]=-x(t) d t+0.5|x(t)|^{\beta} d w_{t}
$$

and

$$
d[x(t)-k x(t)]=2 x(t) d t+|x(t)|^{\beta} d w_{t},
$$

switching among each other according to the movement of the Markov chain $r(t)$. When $\beta \in\left[\frac{1}{2}, 1\right]$, we know that the function $|x(t)|^{\beta}$ is not differentiable and does not satisfy the Lipschitz condition on $[0, T]$. So, we can not prove that Eq.(76) has a unique solution. However, the Eq.(76) satisfies the nonLipschitz condition, then by Theorem 3.1, we have that Eq.(76) has a unique solution.

On the other hand, the Euler approximate solution is defined as follows:

$$
y_{n+1}=\frac{y_{n}}{1-k}+\frac{1}{1-k} a\left(r_{n}^{h}\right) y_{n} h+\frac{1}{1-k} b\left(r_{n}^{h}\right)\left|y_{n}\right|^{\beta} \Delta W_{n},
$$

Here $y_{n} \approx x\left(t_{n}\right)$. Let

$$
\bar{y}(t)=y_{n}, \bar{r}(t)=r_{n}^{h}
$$

for $t \in\left[t_{n}, t_{n+1}\right)$. Then we define the continuous Euler approximate solution $y(t)$ as follows

$\left.y(t)=\frac{1}{1-k} y_{0}+\frac{1}{1-k} \int_{0}^{t} a(\bar{r}(s)) \bar{y}(s) d s+\frac{1}{1-k} \int_{0}^{t} b(\bar{r}(s)) \mid \bar{y}(s)\right)\left.\right|^{\beta} d W_{s} .(78)$ 
Since the Eq.(76) satisfies the conditions of Theorem 4.1, then the approximate solution (78) will converge to the exact solution of Eq.(76) in the mean-square sense.

Remark 5.1 In especially, when $G(r(t), x(t))=0$ and $\beta=\frac{1}{2}$, it will be the hybird square-root process which is the Eq.(1) mentioned above.

Example 5.2 Consider the NSDEs with Markovian switching and pure jumps:

$$
d[x(t)-G(r(t), x(t))]=c(r(t)) \sigma(x(t)) d t+\int_{R^{n}} u \mathcal{H}(x(t)) \tilde{N}(d t, d u),
$$

here the function $G(r(t),$.$) satisfied the condition (H2) and \sigma(v)=\sqrt{|v \log v|}$,

$$
\mathcal{H}(v)= \begin{cases}0, & \text { if } v=0, \\ v \sqrt{\log \left(1+v^{-1}\right)}, & \text { if } 0<v \leq e^{-1}, \\ \frac{\log (1+e)}{e}+\frac{\log (1+e)-1}{2 \sqrt{\log (1+e)}}\left(v-e^{-1}\right), & \text { if } v>e^{-1} .\end{cases}
$$

It is obvious that the coefficient $\sigma($.$) and \mathcal{H}($.$) do not satisfy the Lipschitz$ condition. We have that $\sigma($.$) and \mathcal{H}($.$) are two nondecreasing, positive and$ concave functions on $[0, \infty]$ with $\sigma(0)=\mathcal{H}(0)=0$ and $\int_{0}^{1} \frac{d u}{\sigma(u)}=\int_{0}^{1} \frac{d u}{\mathcal{H}(u)}=$ $\infty$. Therefore, it follows that condition (H1) is satisfied. Consequently, the approximate solution will converge to the exact solution of Eq.(79) for any $t \in[0, T]$ in the sense of Theorem 4.1 .

\section{Acknowledgements}

This work was supported by the National Natural Science Foundation of China (No.11102076), the Natural Science Foundation of Higher Education Institutions of Jiangsu Province (No.10KJD110006), the grant of Jiangsu Institute of Education(No.Jsie2011zd04) and was sponsored by Qing Lan Project of Jiangsu Province (2012).

\section{References}

[1] I.Ya.Kac, N.N.Krasovskii, About stability of systems with stochastic parameters, J.Appl.Math.Mech. 24 (1960) 809-823.

[2] L.E.Shaikhet, Stability of stochastic hereditary systems with Markov switching, Theory.Stoch.Process. 18 (1996) 180-184. 
[3] X.R.Mao,C.G.Yuan, Stochastic Differential Equations with Markovian Switching, Imperial College Press, 2006.

[4] X.R.Mao, Stability of stochastic differential equations with Markovian switching, Stoch.Process.Appl. 79 (1999) 45-67.

[5] C.G.Yuan,X.R.Mao,Asymptotic stability in distribution of stochastic differential equations with Markovian switching,Stoch.Process.Appl. 103 (2003) 277-291.

[6] S.T.Rong, Theory of Stochastic Differential Equations with Jumps and Applications, Mathematical and Analytical Techniques with Applications to Engineering, Dordrecht, Springer,2005.

[7] R.F.Bass,Stochastic differential equations with jumps, Probab.Surveys, 1 (2004) 1-19.

[8] X.Q.Li,C.W.Li,Weak approximations and extrapolations of stochastic diffrential equations with jumps, SIAM J.Numer.Anal. 37 (2000) 1747-1767.

[9] K.Kubilius,E.Platen,Rate of weak convergenece of the Euler approximation for diffusion processes with jumps, Monte.Carlo.Methods.Appl. 8 (2002) 8396.

[10] L.E.Shaikhet, Numerical simulation and stability of stochastic systems with Markovian switching, Neural.Parallel.Sci.Comput. 10 (2002) 199-208.

[11] A.Gardon, The order of approximation for solutions of Ito-type stochastic diffrential equa- tions with jumps, Stoch.Anal.Appl. 22 (2004) 679-699.

[12] C.G.Yuan,X.R.Mao,Convergence of the Euler-Maruyama method for stochastic differential equations with Markovian switching, Math.Comput.Simulat. 64 (2004) 223-235.

[13] X.R.Mao,C.G.Yuan,G.Yin, Numerical method for stationary distribution of stochastic differential equations with Markovian switching, J. Comput. Appl. Math. 174 (2005) 1-27.

[14] X.R.Mao,C.G.Yuan,G.Yin, Approximations of Euler-Maruyama type for stochastic differential equations with Markovian switching under nonLipschitz conditions, J. Comput. Appl.Math. 205 (2007) 936-948.

[15] D.J.Higham,P.E.Kloeden,Numerical methods for nonlinear stochastic differential equations with jumps, Numer.Math. 101 (2005) 101-119. 
[16] D.J.Higham,P.E.Kloeden, Convergence and stability of implicit methods for jump-diffusion systems, Inter.J.Numer.Anal.Model. 3 (2006) 125-140.

[17] D.J.Higham,X.R.Mao,C.G.Yuan, Preserving exponential mean-square stability in the simulation of hybrid stochastic differential equations, Numer.Math. 108 (2007) 295-325.

[18] E.Platen,N.B.Liberati, Numerical Solution of Stochastic Differential Equations with Jumps in Finance, Stoch.Model.Appl.Probab, Springer, 2010.

[19] V.B.Kolmanovskii,V.R.Nosov,Stability of Functional Differential Equations, Academic Press, 1986.

[20] X.R.Mao,Exponential stability in mean square of neutral stochastic differential functional equations, Syst.Control.Lett. 26 (1995) 245-251.

[21] X.R.Mao, Stochastic Differential Equations and Applications, Horwood, New York,1997.

[22] V.Kolmanovskii, N.Koroleva, T.Maizenberg, X.R.Mao, A.Matasov,Neutral stochastic differential delay equations with Markovian switching. Stoch. Anal. Appl. 21 (2003) 819-847.

[23] F.K.Wu, X.R.Mao, Numerical Solutions of Neutral Stochastic Functional Differential Equations, SIAM J.Numer.Anal. 46 (2008) 1821-1841.

[24] S.B.Zhou,F.K.Wu, Convergence of numerical solutions to neutral stochastic delay differential equations with Markovian switching, J.Comput.Appl.Math. 229 (2009) 85-96.

[25] N.Ikeda and S.Watanable, Stochastic Differential Equations and diffusion processes, Oxford, New York,1989.

[26] I.Bihari, A generalization of a lemma of Bellman and its application to uniqueness problem of differential equations, Acta.Math.Acad.Sci.Hungar. 7 (1956) 71-94.

[27] A.V.Skorokhod, Asymptotic Methods in the Theory of Stochastic Differential Equations, Providence, RI: American Mathematical Society, 1989.

[28] P.Billingsley, Convergence of probability measures, JohnWiley and Sons, Inc., New York-London-Sydney, 1968.

[29] W.J.Anderson, Continuous-Time Markov Chains, Springer, Berlin, 1991. 